Honam Mathematical J. 34 (2012), No. 3, pp. 297-310

http://dx.doi.org/10.5831/HMJ.2012.34.3.297

\title{
INTEGRAL POLYNOMIAL SEQUENCES RELATED WITH KRAWTCHOUK MATRICES AND ASSOCIATED RIORDAN ARRAYS
}

\author{
Hyeong-Kwan Ju, HyunJeong Lee and SooJeong Seo
}

\begin{abstract}
The relationship between the integral polynomials obtained from the iterations of the matrix given by the expression (1) and the entries of the Krawtchouk matrices is derived by using the associated Riordan arrays.
\end{abstract}

\section{Introduction}

Lim [7] and Cheon et al. [3] gave questions about the integral polynomial sequences arising in the iterated powers of matrix given by the expression (1) below. In particular, Cheon and Lim [3] showed that certain explicit expressions and combinatorial identities can be presented in terms of Vandermonde matrix, mirrored $\Gamma$-matrix, weighted Delannoy number, Riordan array, hypergeometric function, and Jacobi polynomial, related with the matrix (1). (In fact, one of referees informed authors that there was a manuscript(the reference [3]) regarding the related topics after submitting this paper.) In this article we show that coefficients of the polynomial sequences are nothing but the diagonal elements of the Krawtchouk matrices (which will be introduced in Section 4) and they can be expressed as one of the Riordan arrays. The matrix we consider mostly is

$$
\left(\begin{array}{cc}
1 & 1 \\
x & -x
\end{array}\right)
$$

We also clarify the polynomials that appeared in the iteration of the matrix (1). In other words, we concentrate on the integral polynomials

Received March 15, 2012. Accepted August 8, 2012.

2000 Mathematics Subject Classification. Primary 05A05, 05A10, 05A15; Secondary $11 \mathrm{~B} 65$.

Key words and phrases. Krawtchouk Matrices, Riordan Arrays, Generating Functions. 
$u_{n}(x), v_{n}(x)$ generated by the following :

$$
\left(\begin{array}{cc}
u_{n}(x) & v_{n}(x) \\
z_{n}(x) & w_{n}(x)
\end{array}\right):=\left(\begin{array}{cc}
1 & 1 \\
x & -x
\end{array}\right)^{n+1} \text { for } n=0,1,2, \cdots .
$$

In Section 2 we introduce several basic properties about the polynomials $u_{n}(x)$ and $v_{n}(x)$, some of which will be used later. In Section 3, a Riordan group is defined and we adjust our problem for the Riordan group. A Krawtchouk matrix is introduced and our main results are discussed in Section 4. More detailed results are discussed in Section 5. In the last section further problems are proposed.

\section{Preliminaries}

Polynomials $u_{n}(x)$ and $v_{n}(x)$ given by the formula (2) satisfy the following three-term recursion formulas:

$$
v_{n+2}(x)=(1-x) v_{n+1}(x)+2 x v_{n}(x)
$$

and

$$
u_{n+2}(x)=(1-x) u_{n+1}(x)+2 x u_{n}(x)
$$

for $n=0,1,2, \cdots$. From the recursion formula (3) we can obtain the following formula:

$$
\left(\begin{array}{c}
v_{n+1}(x) \\
v_{n}(x)
\end{array}\right)=\left(\begin{array}{cc}
1-x & 2 x \\
1 & 0
\end{array}\right)^{n}\left(\begin{array}{c}
1-x \\
1
\end{array}\right)=\left(\begin{array}{c}
\frac{\alpha^{n+2}-\beta^{n+2}}{\alpha-\beta} \\
\frac{\alpha^{n+1}-\beta^{n+1}}{\alpha-\beta}
\end{array}\right)
$$

where

$$
\alpha=\frac{1-x+\sqrt{1+6 x+x^{2}}}{2}, \quad \beta=\frac{1-x-\sqrt{1+6 x+x^{2}}}{2}
$$

are two characteristic roots of the matrix $\left(\begin{array}{cc}1-x & 2 x \\ 1 & 0\end{array}\right)$.

It is interesting that from the recurrence formula (3) the ratio of two consecutive polynomials can be expressed as a continued fraction in the 
following:

(5)

$$
\frac{v_{n+1}(x)}{v_{n+2}(x)}=\frac{1}{1-x+\frac{2 x}{1-x+\frac{2 x}{1-x+\frac{2 x}{\ddots \cdot 1-x+\frac{2 x}{1-x}}}}}
$$

Note here that $2 x$ appears $n+1$ times on the righthand side of the equation (5). Likewise,

$$
\frac{u_{n+1}(x)}{u_{n+2}(x)}=\frac{1}{1-x+\frac{2 x}{1-x+\frac{2 x}{1-x+\frac{2 x}{\ddots \cdot 1-x+\frac{2 x}{1+x}}}}}
$$

Note also that $2 x$ appears $n+1$ times on the righthand side of the equation (6).

Using induction we can show that $v_{n}(x)$ and $u_{n}(x)$ are expressed in terms of determinants as in the following associated tridiagonal matrices of the size $(n+1) \times(n+1)$ (the same formulas appeared in $\operatorname{Lim}([7])$ and the Corollary 3.9 of Cheon and $\operatorname{Lim}([3]))$ :

$$
v_{n+1}(x)=\operatorname{det}\left(\begin{array}{cccccc}
1-x & 2 x & 0 & 0 & \cdots & 0 \\
-1 & 1-x & 2 x & 0 & \cdots & 0 \\
0 & -1 & 1-x & 2 x & \cdots & 0 \\
\vdots & \vdots & \ddots & \ddots & \ddots & \vdots \\
0 & 0 & \cdots & -1 & 1-x & 2 x \\
0 & 0 & \cdots & 0 & -1 & 1-x
\end{array}\right)
$$

and

$$
u_{n+1}(x)=\operatorname{det}\left(\begin{array}{cccccc}
1-x & 2 x & 0 & 0 & \cdots & 0 \\
-1 & 1-x & 2 x & 0 & \cdots & 0 \\
0 & -1 & 1-x & 2 x & \cdots & 0 \\
\vdots & \vdots & \ddots & \ddots & \ddots & \vdots \\
0 & 0 & \cdots & -1 & 1-x & 2 x \\
0 & 0 & \cdots & 0 & -1 & 1
\end{array}\right)
$$




\section{Riordan Arrays}

A Riordan group is a set of lower triangular integer matrices $\left(d_{i, j}\right)$ of infinite size (called a Riordan array) so that each matrix satisfies the following (i)-(iii):

(i) $p(t)=p_{0}+p_{1} t+p_{2} t^{2}+\cdots$,

(ii) $q(t)=q_{1} t+q_{2} t^{2}+q_{3} t^{3}+\cdots=\operatorname{th}(t)\left(q_{1}=h(0) \neq 0\right)$, and

(iii) $p(t) q(t)^{k}=\sum_{i=0}^{\infty} d_{i, k} t^{i}$.

Thus, an alternative form of the Riordan group is the set of all pairs $(p(t), q(t))$ of such functions $p(t)$ and $q(t)$ under the operation $\bullet$ which is described as in the following:

For any two pairs $(p, q)$ and $(r, s)$ in the set,

$$
(p, q) \bullet(r, s)=(p(r \circ q), s \circ q) .
$$

where $(f \circ g)(t)=f(g(t))$.

The identity pair is $(1, t)$ and $(p, q)^{-1}=\left(\frac{1}{p \circ q^{-1}}, q^{-1}\right)$. (See [2] for details.) To each Riordan array $(p, q)=(p, t h)$ there is an integer sequence $A=\left\{a_{i}\right\}$ with $a_{0} \neq 0$ satisfying

$$
d_{n+1, k+1}=a_{0} d_{n, k}+a_{1} d_{n, k+1}+a_{2} d_{n, k+2}+\cdots \quad(n, k \geq 0) .
$$

where $d_{i j}$ is the $(i, j)$ th entry of the Riordan array $(p, q)$. Such a sequence $A=\left\{a_{i}\right\}$ is called the $\mathbf{A}$ - sequence of the Riordan array $(p, q)$. We can find the generating function $A(t)=\sum_{n=o}^{\infty} a_{n} t^{n}$ from the equation $A(t h(t))=h(t)$. A Riordan array of the form $(p(t), t)$ (where $p(t)=$ $\left.\sum_{n=o}^{\infty} b_{n} t^{n}\right)$ is called the Appell array of the sequence $\left\{b_{n}\right\}$. Its $(n, k)$ th entry of the Riordan array $(p(t), t)$ is $b_{n-k}$. We realize immediately that

$$
\sum_{n=o}^{\infty} \sum_{k=o}^{\infty} d_{n, k} t^{n} x^{k}=\frac{p(t)}{1-x q(t)}
$$

Now, back to the recursive formula (3), we can find that its generating function is as follows:

$$
G(x, t):=\sum_{k=o}^{\infty} v_{n}(x) t^{n}=\frac{\frac{1}{1-t}}{1-x \frac{t(2 t-1)}{1-t}}=\frac{p(t)}{1-x q(t)} .
$$

We denote the Riordan array $(p(t), q(t))=\left(\frac{1}{1-t}, \frac{t(2 t-1)}{1-t}\right)$ by $\left\{t_{n, k}\right\}$. In fact, a similar Riordan array appeared in the Proposition 7 of Barry([2]). 


\begin{tabular}{l|lllllllllll} 
& $\mathrm{k}=0$ & 1 & 2 & 3 & 4 & 5 & 6 & 7 & 8 & 9 & 10 \\
\hline $\mathrm{n}=0$ & 1 & & & & & & & & & & \\
1 & 1 & -1 & & & & & & & & & \\
2 & 1 & 0 & 1 & & & & & & & & \\
3 & 1 & 1 & -1 & -1 & & & & & & & \\
4 & 1 & 2 & -2 & 2 & 1 & & & & & & \\
5 & 1 & 3 & -2 & 2 & -3 & -1 & & & & & \\
6 & 1 & 4 & -1 & 0 & -1 & 4 & 1 & & & & \\
7 & 1 & 5 & 1 & -3 & 3 & -1 & -5 & -1 & & & \\
8 & 1 & 6 & 4 & -6 & 6 & -6 & 4 & 6 & 1 & & \\
9 & 1 & 7 & 8 & -8 & 8 & -6 & 8 & -8 & -7 & -1 & \\
10 & 1 & 8 & 13 & -8 & 2 & 0 & 2 & -8 & 13 & 8 & 1
\end{tabular}

Figure 1. Table for $\left\{t_{n, k}\right\}$

(See Figure 1 for the table regarding the sequence $\left(t_{n, k}\right)$.) Thus,

$$
v_{n}(x)=\sum_{k=o}^{\infty} t_{n, k} x^{k}
$$

and the generating function associated with A-sequence is

$$
\begin{aligned}
A(x)= & \frac{x-1-\sqrt{1+6 x+x^{2}}}{2} \\
=(-1) & +(-1) x+2 x^{2}+(-6) x^{3}+22 x^{4}+(-90) x^{5}+394 x^{6} \\
& +(-1806) x^{7}+8558 x^{8}+(-41586) x^{9}+\cdots .
\end{aligned}
$$

This is a sequence which appeared in OEIS([8]) (id: A103137, a first column of inverse Delannoy triangle). Its signless sequence is A006318, large Schroeder numbers, in OEIS([8]).

From the definition of the A-sequence we get a recursive formula for the sequence $\left\{t_{n k}\right\}$ as follows:

$t_{n+1, k+1}=(-1) t_{n, k}+(-1) t_{n, k+1}+2 t_{n, k+2}+(-6) t_{n, k+3}+22 t_{n, k+4}+\cdots$

Similarly, the generating function for the polynomial sequence $\left\{u_{n}(x)\right\}$ is given by:

$$
H(x, t):=\sum_{n=o}^{\infty} u_{n}(x) t^{n}=\frac{\frac{1}{1-t}}{1-x \frac{t(2 t-1)}{1-t}}+2 x t \frac{\frac{1}{1-t}}{1-x \frac{t(2 t-1)}{1-t}} .
$$


Let $\left\{s_{n, k}\right\}$ be the infinite lower-triangular matrix associated with the polynomial sequence $\left\{u_{n}(x)\right\}$. Then,

$$
u_{n}(x)=s_{n, 0}+s_{n, 1} x+\cdots+s_{n, n} x^{n}(n \geq 0) .
$$

From the formula (8), the relation between $\left\{s_{n, k}\right\}$ and $\left\{t_{n, k}\right\}$ satisfies the following:

$$
s_{n+1, k+1}=t_{n+1, k+1}+2 t_{n, k} \quad(n, k \geq 0),
$$

which implies that

$$
u_{n+1}(x)=v_{n+1}(x)+2 x v_{n}(x) .
$$

Likewise, for the polynomial sequence $\left\{z_{n}(x)\right\}$,

$$
J(x, t):=\sum_{n=0}^{\infty} z_{n}(x) t^{n}=\frac{2 x t-x}{1-t+x t-2 x t^{2}}=2 x t \frac{\frac{1}{1-x}}{1-x \frac{t(2 t-1)}{1-t}}-x \frac{\frac{1}{1-t}}{1-x \frac{t(2 t-1)}{1-t}} .
$$

Let $\left\{p_{n, k}\right\}$ be the infinite lower-triangular matrix associated with the polynomial sequence $\left\{z_{n}(x)\right\}$. Then, $z_{n}(x)=p_{n, 0}+p_{n, 1} x+\cdots+p_{n, n} x^{n}+$ $p_{n, n+1} x^{n+1}$ and $p_{n+1, k+1}=-t_{n+1, k}+2 t_{n, k} \quad(n, k \geq 0)$. As above, $z_{n+1}(x)=-x v_{n+1}(x)+2 x v_{n}(x)$.

Now, for the polynomial $\left\{w_{n}(x)\right\}$,

$$
K(x, t):=\sum_{n=0}^{\infty} w_{n}(x) t^{n}=\frac{x}{1-t+x t-2 x t^{2}}=x \frac{\frac{1}{1-x}}{1-x \frac{t(2 t-1)}{1-t}}=x \frac{\frac{1}{1-x}}{1-x \frac{t(2 t-1)}{1-t}} .
$$

Let $\left\{q_{n, k}\right\}$ be the infinite lower-triangular matrix associated with the polynomial sequence $\left\{w_{n}(x)\right\}$. Then, $w_{n}(x)=q_{n, 0}+q_{n, 1} x+\cdots+q_{n, n} x^{n}+$ $q_{n, n+1} x^{n+1}$ and $q_{n, k+1}=t_{n, k}(n, k \geq 0)$. This says that $w_{n}(x)=x v_{n}(x)$.

In summary, we have

$$
\begin{array}{rlrl}
u_{n+1}(x) & =v_{n+1}(x)+2 x v_{n}(x) \\
z_{n+1}(x) & = & -x v_{n+1}(x)+2 x v_{n}(x) \\
w_{n+1}(x) & = & x v_{n+1}(x) .
\end{array}
$$




\section{Krawtchouk Matrices}

For details about Krawtchouk matrices, see [4] or the references therein. The n-th order Krawtchouk matrix $K^{(n)}=\left(K_{i j}^{(n)}\right)$ is a matrix of size $(n+1) \times(n+1)$ whose entries satisfy the following :

$$
(1+x)^{n-j}(1-x)^{j}=\sum_{i=0}^{n} x^{i} K_{i j}^{(n)}
$$

From the formula (10), we can express each entry explicitly as follows:

$$
K_{i j}^{(n)}=\sum_{k \geq 0}(-1)^{k}\left(\begin{array}{l}
j \\
k
\end{array}\right)\left(\begin{array}{c}
n-j \\
i-k
\end{array}\right)
$$

Hence $K_{i i}^{(n)}$, the $i-t h$ element of the diagonal in the $n-t h$ order Krawtchouk matrix $K^{(n)}$, is

$$
K_{i i}^{(n)}=\sum_{k \geq 0}(-1)^{k}\left(\begin{array}{c}
i \\
k
\end{array}\right)\left(\begin{array}{c}
n-i \\
i-k
\end{array}\right) \quad(0 \leq i \leq n)
$$

As a reference we list first several $(0 \leq n \leq 4)$ Krawtchouk matrices below.

$\left(\begin{array}{l}1\end{array}\right),\left(\begin{array}{cc}1 & 1 \\ 1 & -1\end{array}\right),\left(\begin{array}{ccc}1 & 1 & 1 \\ 2 & 0 & -2 \\ 1 & -1 & 1\end{array}\right),\left(\begin{array}{cccc}1 & 1 & 1 & 1 \\ 3 & 1 & -1 & -3 \\ 3 & -1 & -1 & 3 \\ 1 & -1 & 1 & -1\end{array}\right),\left(\begin{array}{ccccc}1 & 1 & 1 & 1 & 1 \\ 4 & 2 & 0 & -2 & -4 \\ 6 & 0 & -2 & 0 & 6 \\ 4 & -2 & 0 & 2 & -4 \\ 1 & -1 & 1 & -1 & 1\end{array}\right)$.

THEOREM 1 For every nonnegative integer n,

$$
v_{n}(x)=\sum_{i=0}^{n} K_{i i}^{(n)} x^{i}
$$

In order to prove Theorem 1 we need the following Lemma which is described in [5].(See the formula (5.25) on page 169 of the reference [5].)

LEMMA 2

$$
\sum_{k \geq 0}\left(\begin{array}{c}
l \\
m+k
\end{array}\right)\left(\begin{array}{c}
s+k \\
n
\end{array}\right)(-1)^{k}=\left(\begin{array}{c}
s-m \\
n-l
\end{array}\right)(-1)^{l+m} .
$$


Proof of the THEOREM 1:

$$
\begin{aligned}
& {\left[x^{i}\right]\left(v_{n}(x)\right)=t_{n, i}=\left[t^{n}\right]\left(\frac{1}{1-t}\left(\frac{t(2 t-1)}{1-t}\right)^{i}\right)} \\
& =\left[t^{n-i}\right]\left(\frac{(2 t-1)^{i}}{(1-t)^{i+1}}\right) \\
& =\left[t^{n-i}\right]\left(\sum_{k=0}^{i}\left(\begin{array}{l}
i \\
k
\end{array}\right)(2 t)^{k}(-1)^{i-k} \sum_{l=0}^{\infty}\left(\begin{array}{c}
i+l \\
l
\end{array}\right) t^{l}\right) \\
& =\sum_{k=0}^{i}\left(\begin{array}{l}
i \\
k
\end{array}\right) 2^{k}(-1)^{i-k}\left(\begin{array}{c}
n-k \\
i
\end{array}\right) \\
& =\sum_{k=0}^{i}\left(\begin{array}{c}
n-k \\
i
\end{array}\right)\left(\begin{array}{l}
i \\
k
\end{array}\right)(-1)^{i-k} \sum_{m=0}^{k}\left(\begin{array}{c}
k \\
m
\end{array}\right) \\
& =\sum_{m=0}^{i} \sum_{k=m}^{i}\left(\begin{array}{l}
i \\
k
\end{array}\right)\left(\begin{array}{c}
k \\
m
\end{array}\right)\left(\begin{array}{c}
n-k \\
i
\end{array}\right)(-1)^{i-k} \\
& =\sum_{m=0}^{i} \sum_{k=m}^{i}\left(\begin{array}{c}
i \\
m
\end{array}\right)\left(\begin{array}{c}
i-m \\
k-m
\end{array}\right)\left(\begin{array}{c}
n-k \\
i
\end{array}\right)(-1)^{i-k} \\
& =\sum_{m=0}^{i}\left(\begin{array}{c}
i \\
m
\end{array}\right)(-1)^{i} \sum_{k=m}^{i}\left(\begin{array}{c}
i-m \\
k-m
\end{array}\right)\left(\begin{array}{c}
n-k \\
i
\end{array}\right)(-1)^{k} \\
& =\sum_{m=0}^{i}\left(\begin{array}{c}
i \\
m
\end{array}\right) \sum_{p=0}^{i-m}\left(\begin{array}{c}
i-m \\
p
\end{array}\right)\left(\begin{array}{c}
p+n-i \\
i
\end{array}\right)(-1)^{p} \quad(p:=i-k) \\
& =\sum_{m=0}^{i}\left(\begin{array}{c}
i \\
m
\end{array}\right)\left(\begin{array}{c}
n-i \\
m
\end{array}\right)(-1)^{i-m} \quad \text { (by Lemma2) } \\
& =\sum_{k=0}^{i}\left(\begin{array}{l}
i \\
k
\end{array}\right)\left(\begin{array}{l}
n-i \\
i-k
\end{array}\right)(-1)^{k} \quad(k:=i-m) \\
& =K_{i i}^{(n)} \text { (by the formula (12)) }
\end{aligned}
$$

From the formula (12) we also see that

$$
K_{n-i, n-i}^{(n)}=(-1)^{n} K_{i i}^{(n)},
$$

and, therefore (from Theorem 1) we have 


$$
v_{n}(x)=(-x)^{n} v_{n}\left(\frac{1}{x}\right) .
$$

Equation (13) says that $v_{n}(x)$ is a reciprocal (resp. anti-reciprocal) polynomial if $\mathrm{n}$ is even (resp. odd). Hence $x$ is a zero of $v_{n}$ if and only if $\frac{1}{x}$ is a zero of $v_{n}$.

\section{COROLLARY 3}

(1) Trace of the (2n)th order Krawtchouk matrix $K^{(2 n)}$ is $2^{n}$, and

$$
\sum_{m=0}^{2 n} \sum_{k=0}^{m}(-1)^{k}\left(\begin{array}{c}
m \\
k
\end{array}\right)\left(\begin{array}{c}
2 n-m \\
m-k
\end{array}\right)=2^{n} .
$$

(2) Trace of the $(2 n+1)$ st order Krawtchouk matrix $K^{(2 n+1)}$ is 0 .

$$
\sum_{m=0}^{2 n+1} \sum_{k=0}^{m}(-1)^{k}\left(\begin{array}{c}
m \\
k
\end{array}\right)\left(\begin{array}{c}
2 n+1-m \\
m-k
\end{array}\right)=0 .
$$

Proof: It is obvious from $v_{2 n}(1), v_{2 n+1}(1)$ and formula (3).

In the next Theorem we describe coefficients of $u_{n}(x)$.

\section{THEOREM 4}

$$
u_{n}(x)=K_{00}^{(n)}+\sum_{k=1}^{n} K_{k, k-1}^{(n)} x^{k} .
$$

Note that this result says that the constant term of $u_{n}(x)$ is $u_{n}(0)=$ $1=K_{00}^{(n)}$ and the rest of its coefficients consist of subdiagonal entries of the $n$th order Krawtchouk matrix $K^{(n)}=\left(K_{i j}^{(n)}\right)$.

Proof: It is obvious that $u_{n}(0)=1=K_{00}^{(n)}$. Since the coefficients of

$$
u_{n}(x)=\sum_{k=o}^{n} s_{n k} x^{k}
$$

satisfies the formula (9) and $t_{n i}=K_{i i}^{(n)}$ (Theorem 1), it is enough to show that for $n=0,1,2, \cdots$ and $k=0,1,2, \cdots, n$. 


\begin{tabular}{l|lllllllll}
$\mathrm{n} / \mathrm{k}$ & 0 & 1 & 2 & 3 & 4 & 5 & 6 & 7 & 8 \\
\hline 0 & 1 & & & & & & & & \\
1 & 1 & -1 & & & & & & & \\
2 & -1 & 0 & 1 & & & & & & \\
3 & 3 & -1 & -1 & -1 & & & & & \\
4 & -11 & 4 & 4 & 2 & 1 & & & & \\
5 & 45 & -17 & -16 & -8 & -3 & -1 & & & \\
6 & -197 & 76 & 69 & 34 & 13 & 4 & 1 & & \\
7 & 903 & -353 & -313 & -153 & -59 & -19 & -5 & -1 & \\
8 & -4279 & 1688 & 1472 & 716 & 273 & 92 & 26 & 6 & 1
\end{tabular}

Figure 2. Table for $\left\{s_{n, k}\right\}$

$$
\begin{aligned}
K_{k+1, k}^{(n+1)} & =\sum_{i \geq 0}(-1)^{i}\left(\begin{array}{c}
k \\
i
\end{array}\right)\left(\begin{array}{c}
n+1-k \\
k+1-i
\end{array}\right) \\
& =\sum_{i \geq 0}(-1)^{i}\left(\begin{array}{c}
k \\
i
\end{array}\right)\left(\begin{array}{c}
n-k \\
k+1-i
\end{array}\right)+\sum_{i \geq 0}(-1)^{i}\left(\begin{array}{c}
k \\
i
\end{array}\right)\left(\begin{array}{c}
n-k \\
k-i
\end{array}\right) \\
& =\sum_{i \geq 0}(-1)^{i}\left[\left(\begin{array}{c}
k+1 \\
i
\end{array}\right)-\left(\begin{array}{c}
k \\
i-1
\end{array}\right)\right]\left(\begin{array}{c}
n-k \\
k+1-i
\end{array}\right)+K_{k k}^{(n)} \\
& =K_{k+1, k+1}^{(n+1)}+2 K_{k k}^{(n)}
\end{aligned}
$$

Note that

$$
\begin{gathered}
\left(\frac{1}{1-x}, \frac{x(2 x-1)}{1-x}\right)^{-1}=\left(\frac{3+x+\sqrt{1+6 x+x^{2}}}{4}, \frac{1-x-\sqrt{1+6 x+x^{2}}}{4}\right)=:(\beta(x), \gamma(x)) \\
\frac{\beta(x)}{1-t \gamma(x)}=\frac{3+x+\sqrt{1+6 x+x^{2}}}{4-t(1-x)+t \sqrt{1+6 x+x^{2}}},
\end{gathered}
$$

From $A(\gamma(x))=\frac{\gamma(x)}{x}, A(x)=\frac{1-x}{2 x-1}$.

Table for the sequence $\left\{s_{n, k}\right\}$ is given for reference in Figure 2. The 0th column of Figure 2 has an id A080243(and its signless version A001003) in [8]. Each row sum is zero since $\frac{\beta(x)}{1-\gamma(x)}=1$. Thus the signless numbers in the 0th column (called Super- Catalan numbers or Little Schröder numbers) are decomposed of numbers in the rest of the columns.

Anti-diagonal entries of the $n$ th-order Krawtchouk matrix $K^{(n)}=\left(K_{i j}^{(n)}\right)$ 
can give us other integral polynomials, such as

$$
s_{n}(x)=\sum_{k=0}^{n} K_{k, n-k}^{(n)} \quad(n \geq 0) .
$$

$s_{n}(x)$ is a reciprocal polynomial since $K_{k, n-k}^{(n)}=K_{n-k, k}^{(n)}$. Its Riordan array given by the lower triangular matrix $\left\{r_{i j}\right\}$ of infinite size is $(p(x),-q(x))=\left(\frac{1}{1-x}, \frac{x(1-2 x)}{1-x}\right)$ which is described in [2].(See also id A098593 in [8].)

Note that

$$
\begin{gathered}
\left(\frac{1}{1-x}, \frac{x(1-2 x)}{1-x}\right)^{-1}=\left(\frac{1-x+\sqrt{1-6 x+x^{2}}}{2}, \frac{1+x-\sqrt{1-6 x+x^{2}}}{2}\right)=(2 \beta(-x)-1,2 \gamma(-x)) \\
\frac{2 \beta(-x)-1}{1-t(2 \gamma(-x))}=\frac{1-x+\sqrt{1-6 x+x^{2}}}{2-t(1+x)+t \sqrt{1-6 x+x^{2}}} . \\
A(x)=\frac{x-2}{1-x} .
\end{gathered}
$$

\section{Further Details}

In the proof of Theorem 4 we have shown that a subdiagonal element $K_{k+1, k}^{(n+1)}$ of the $(n+1)$ st Krawtchouk matrix can be expressed in terms of the diagonal elements of the $n$th and $(n+1)$ st Krawtchouk matrices. That is,

$$
K_{k+1, k}^{(n+1)}=K_{k+1, k+1}^{(n+1)}+2 K_{k k}^{(n)}(0 \leq k \leq n) .
$$

Likewise, we can get the similar formula for $K_{k+2, k}^{(n+2)}$ as following:

(14) $K_{k+2, k}^{(n+2)}=K_{k+2, k+2}^{(n+1)}+3 K_{k+1, k+1}^{(n+1)}+2 K_{k+1, k+1}^{(n)}+4 K_{k k}^{(n)}(0 \leq k \leq n)$.

Note here that the value of $K_{a, a}^{b}$ with $a>b$ is zero. For example,

$$
K^{(2)}=\left(\begin{array}{ccc}
(1) & 1 & 1 \\
2 & (0) & -2 \\
1 & -1 & (1)
\end{array}\right), K^{(3)}=\left(\begin{array}{cccc}
(1) & 1 & 1 & 1 \\
3 & (1) & -1 & -3 \\
3 & -1 & (-1) & 3 \\
1 & -1 & 1 & (-1)
\end{array}\right)
$$


$K_{2,0}^{(4)}=K_{2,2}^{(3)}+3 K_{1,1}^{(3)}+2 K_{1,1}^{(2)}+4 K_{0,0}^{(2)}=(-1)+3(1)+2(0)+4(1)=[6]$.

$K_{4,2}^{(4)}=K_{4,4}^{(3)}+3 K_{3,3}^{(3)}+2 K_{3,3}^{(2)}+4 K_{2,2}^{(2)}=(0)+3(-1)+2(0)+4(1)=<1>$.

In general, identity (14) holds because:

$$
\begin{aligned}
K_{k+2, k}^{(n+2)}= & \sum_{i \geq 0}(-1)^{i}\left(\begin{array}{c}
k \\
i
\end{array}\right)\left(\begin{array}{c}
n+2)-k \\
(k+2)-i
\end{array}\right) \\
= & \sum_{i \geq 0}(-1)^{i}\left(\begin{array}{c}
k \\
i
\end{array}\right)\left(\begin{array}{c}
n+1-k \\
k+2-i
\end{array}\right)+\sum_{i \geq 0}(-1)^{i}\left(\begin{array}{c}
k \\
i
\end{array}\right)\left(\begin{array}{c}
n+1-k \\
k+1-i
\end{array}\right) \\
= & \sum_{i \geq 0}(-1)^{i}\left[\left(\begin{array}{c}
k+1 \\
i
\end{array}\right)-\left(\begin{array}{c}
k \\
i-1
\end{array}\right)\right]\left(\begin{array}{c}
n+1-k \\
k+2-i
\end{array}\right)+K_{k+1, k+1}^{(n+1)}+2 K_{k, k}^{(n)} \\
= & \sum_{i \geq 0}(-1)^{i}\left(\begin{array}{c}
k+1 \\
i
\end{array}\right)\left(\begin{array}{c}
n+1-k \\
k+2-i
\end{array}\right)-\sum_{i \geq 0}(-1)^{i}\left(\begin{array}{c}
k \\
i-1
\end{array}\right)\left(\begin{array}{c}
n+1-k \\
k+2-i
\end{array}\right)+K_{k+1, k+1}^{(n+1)}+2 K_{k, k}^{(n)} \\
= & \sum_{i \geq 0}(-1)^{i}\left(\begin{array}{c}
k+1 \\
i
\end{array}\right)\left(\begin{array}{c}
(n+2)-(k+1) \\
(k+1)+1-i
\end{array}\right)+\sum_{l \geq 0}(-1)^{l}\left(\begin{array}{c}
k \\
l
\end{array}\right)\left(\begin{array}{c}
n+1-k \\
k+1-l
\end{array}\right)+K_{k+1, k+1}^{(n+1)}+2 K_{k, k}^{(n)} \\
= & \sum_{i \geq 0}(-1)^{i}\left(\begin{array}{c}
k+1 \\
i
\end{array}\right)\left\{\left(\begin{array}{c}
n+1-(k+1) \\
(k+1) 1-i
\end{array}\right)+\left(\begin{array}{c}
n+1-(k+1) \\
(k+1)-i
\end{array}\right)\right\}+2\left(K_{k+1, k+1}^{(n+1)}+2 K_{k, k}^{(n)}\right) \\
= & \left(K_{k+2, k+2}^{(n+1)}+2 K_{k+1, k+1}^{(n)}\right)+K_{k+1, k+1}^{(n+1)}+2\left(K_{k+1, k+1}^{(n+1)}+2 K_{k, k}^{(n)}\right) \\
= & K_{k+2, k+2}^{(n+1)}+2 K_{k+1, k+1}^{(n)}+K_{k+1, k+1}^{(n+1)}+2\left(K_{k+1, k+1}^{(n+1)}+2 K_{k, k}^{(n)}\right) \\
= & K_{k+2, k+2}^{(n+1)}+3 K_{k+1, k+1}^{(n+1)}+2 K_{k+1, k+1}^{(n)}+4 K_{k, k}^{(n)}
\end{aligned}
$$

For $n=0,1,2, \cdots$, we consider the polynomial $\rho_{n+2}(x)$ of degree $n+2$,

$$
\rho_{n+2}(x):=K_{0,0}^{(n+2)}+K_{1,0}^{(n+2)} x+\sum_{k=0}^{n} K_{k+2, k}^{(n+2)} x^{k+2} .
$$

Let $\rho_{0}(x)=1, \rho_{1}(x)=1+x$. By the previous definition (15), for instance, $\rho_{2}(x)=1+2 x+x^{2}, \rho_{3}(x)=1+3 x+3 x^{2}-x^{3}, \rho_{4}(x)=1+4 x+6 x^{2}-$ $2 x^{3}+x^{4}$. Actually, this comes from the fact that

$$
\rho_{n+2}(x)=(1+3 x) v_{n+1}(x)+2 x(1+2 x) v_{n}(x)-2 x,
$$

which can be obtained using (14) and (15). By the formula (16) and the recurrence relation $(3)$ on $v_{n}(x)$ we get the following recurrence relation on $\rho_{n}(x)$ :

$$
\rho_{n+2}(x)=(1-x) \rho_{n+1}(x)+2 x \rho_{n}(x)+2 x^{2} .
$$


If we let $M(x, t):=\sum_{n=0}^{\infty} \rho_{n}(x) t^{n}$ then from the recursion (17) we obtain the following:

$$
M(x, t)=\frac{1+2 x t+\frac{2 x^{2} t^{2}}{1-t}}{1-t-x\left(2 t^{2}-t\right)}=\frac{\frac{1}{1-t}}{1-x \frac{t(2 t-1)}{1-t}}+2 x t \frac{\frac{1}{1-t}}{1-x \frac{t(2 t-1)}{1-t}}+2 x^{2} t^{2} \frac{\frac{1}{(1-t)^{2}}}{1-x \frac{t(2 t-1)}{1-t}}
$$

\section{Proposed Problems}

1. For a function

$$
f_{n}(x)=\frac{(2 x-1)^{n}}{(1-x)^{n+1}},
$$

is it true that $\frac{d^{k} f_{n}}{d x^{k}}(0)$ is nonzero for $k=0,1,2, \cdots, n-1$ ? In particular, we can see that

$$
\frac{d^{2 k+1} f_{2 k+1}}{d x^{2 k+1}}(0)=0 \text { and } \frac{d^{2 k} f_{2 k}}{d x^{2 k}}(0) /(2 k) !=(-1)^{k}\left(\begin{array}{c}
2 k \\
k
\end{array}\right)
$$

for $k \geq 0$ by the similar way as in the proof of Theorem 1 . It seems that the problem posed here has certain significance in the reconstruction problem related with the $(7,4,3)$-Hamming code $([6]$ and $[1])$.

2. Characterize the roots of the reciprocal functions or anti-reciprocal functions $v_{n}(x)$. In particular, can we describe the relative positions of the roots of reciprocal functions or anti-reciprocal functions $v_{n}(x)$ ?

3. Can we show those identity formulas given in the Corollary 3 combinatorially?

4. Generalize the problem that we pose. That is, consider iterations of the matrix

$$
\left(\begin{array}{cc}
1 & 1 \\
s x & t x
\end{array}\right)
$$

More generally, is it possible to translate this problem into any $q$-analog version of that and obtain the associated results?

5. Generalize the formula (14). In other words, find the general formula for $K_{k+i, k}^{(n+2)}(i \geq 1,0 \leq k \leq n)$ in terms of diagonal elements of Krawtchouk matrices of the smaller size.

Acknowledgements The authors would like to give their thanks to Prof. Yongdo Lim for introducing this interesting topic. The authors would also like to express their thanks to the referees for their comments and, in particular, one of the referees for providing the reference ([3]). 
One of the authors $(\mathrm{Ju})$ would like to extends his gratitude to Ms. Tayler Flory for reviewing.

\section{References}

[1] Sergey V. Avgustinovich, On a property of perfect binary codes, (Russian)Diskret.Anal.Issled.Oper. vol.2(1995), no.1, 4-6.

[2] Paul Barry, A note on Krawtchouk polynomials and Riordan arrays, J. of Integer Sequences, vol.11(2008) Article 08.2.2, 1-16.

[3] Gi-Sang Cheon and Yongdo Lim, Integral polynomial sequences arising from the matrix powers of order 2, to appear in Linear Algebra and Its Applications.

[4] Phillip Feinsilver and Jerzy Kocik, Krawtchouk polynomials and Krawtchouk matrices, Recent Advances in Applied Probability, Spring-Verlag, October, 2004. See also http://aps.arxiv.org/abs/quant-ph/0702073.

[5] Ronald Graham, Donald Knuth and Oren Patashnik, Concrete Mathematics, Addison-Wesley, 1994.

[6] Jong Yoon Hyun, Private communication.

[7] Yongdo Lim, Integral polynomial sequences from weighted Delannoy numbers, materials presented at Chonnam National University on August 2010.

[8] On-Line Encyclopedia of Integer Sequences, available at http://oeis.org/

\section{Hyeong-Kwan Ju}

Department of Mathematics, Chonnam National University

Gwangju, 500-757, Korea

E-mail: hkju@chonnam.ac.kr

HyunJeong Lee

Department of Mathematics, Chonnam National University

Gwangju, 500-757, Korea

E-mail: did-2@hanmail.net

\section{SooJeong Seo}

Department of Mathematics, Chonnam National University

Gwangju, 500-757, Korea

E-mail: yahwoo98@gmail.com 